American Journal of Infectious Diseases 3 (3): 128-133, 2007

ISSN 1553-6203

(C) 2007 Science Publications

\title{
Standard versus Specific Therapy for Chronic Osteomyelitis Treatment
}

\author{
${ }^{1}$ Angela Ingianni, ${ }^{1}$ Giorgio Lampis, ${ }^{1,3}$ Samuela Laconi, ${ }^{2}$ Donatina Cariello, and ${ }^{1,3}$ Raffaello Pompei \\ ${ }^{1}$ Department of Biomedical Sciences and Technology, Cattedra di Microbiologia Applicata, Facoltà di \\ Farmacia, via Porcell 4, 09124 Cagliari; \\ ${ }^{2}$ Clinica Ortopedica II, Università di Pisa, Italy; \\ ${ }^{3}$ Biotecne, Cagliari, Italy
}

\begin{abstract}
: 58 patients with chronic osteomyelitis were treated either with a standard therapy or with a specific targeted therapy based on the antibiogram assay. Standard therapy was performed by a 15 -day course of a parenteral cephalosporin (usually ceftriaxone) in combination with an aminoglycoside (e.g. netilmicin), followed by oral therapy with a fluoroquinolone (generally ciprofloxacin) for 1 to 3 months; specific therapy largely varied depending on the antibiogram response. The results indicated that no significant differences were found between the patients who received standard therapy $(95.5 \%$ cured) and those who received a specific therapy (93.5\% cured), after a one year follow-up. It can be concluded that a short-term combined parenteral-oral standard therapy, indicated in all those cases where antibiotic therapy must be started before obtaining the laboratory response, or when a clear and definite identification of the microorganisms involved in the infection is not possible, can be as efficient as an antibiogram-guided therapy in the treatment of chronic osteomyelitis.
\end{abstract}

Key words: Chronic osteomyelitis; Standard therapy; Quinolones; Ceftriaxone; Staphylococci; Microbial resistance

\section{INTRODUCTION}

Chronic osteomyelitis has been defined as a recalcitrant disease, because it sometimes shows marked difficulties in response to the antibiotic treatment ${ }^{[1,2]}$. This is due to some particular situations that characterize this type of infection; firstly, the drugs' difficulty of access into the site of the infection ${ }^{[3-7]}$; then, the frequent presence of multi-drug resistant bacteria ${ }^{[8,9]}$ and finally, problems in the identification of the real etiologic agent of the disease ${ }^{[10,11]}$.

A large portion of osteomyelitis in adults is chronic. Chronic osteomyelitis is defined by the presence of a draining sinus, prolonged disease duration and disease resistance to a course of antimicrobial therapy ${ }^{[2]}$. Chronic osteomyelitis in adults is also a disease associated with frequent therapeutic failures. The most rational diagnosis of chronic osteomyelitis is based on the presence of infected necrotic bone with an envelope of infected soft tissue requiring operative debridement. Chronic osteomyelitis was defined by Mader et al. ${ }^{[11]}$ as a disease with a long-lasting symptomatology (more than 10 days).

Several antibiotic regimens have been proposed for chronic osteomyelitis treatment. The first antibiotics, which showed a really good therapeutic efficacy, were beta-lactams, (mainly the newer cephalosporins), used alone or in combination with other drugs ${ }^{[1,12]}$. Third generation cephalosporins are effective, but they are mainly indicated for an ambulatory treatment of Staphylococcus aureus osteomyelitis ${ }^{[1,11,12]}$. More recently great interest has been shown in the use of fluoroquinolones, because they can be taken by the oral route, are well tolerated for long-regimen treatments, have a good bone distribution and a rapid efficacy on many gram positive and negative bacteria. ${ }^{[2,9,13]}$. The efficacy of these treatments ranged from $65 \%{ }^{[14]}$ to $95 \%{ }^{[15]}$

Oral quinolones are commonly used in the treatment of osteomyelitis ${ }^{[7,13-22]}$. Moreover Greenberg et al. ${ }^{[20]}$ claimed that they are safe and effective if they are given for a prolonged course of treatment for infections caused by susceptible gram-positive as well 
as gram-negative organisms and in combination with adequate surgical debridement.

Lew \& Waldvogel ${ }^{[7]}$ reported a 90\% clinical success with ciprofloxacin and ofloxacin in osteomyelitis caused by enterobacteria. These authors conclude that further comparative studies, using quinolones as single agents or in combination (compared to standard parenteral therapy), are necessary on osteomyelitis due to Staphylococcus aureus and Pseudomonas aeruginosa. However, they stress the finding that quinolones have several advantages over other traditional compounds in the therapy for osteomyelitis or for orthopaedic prosthetic device infections because: a) after an initial course of intravenous therapy they can be administered orally with excellent bioavailability; b) they are relatively non-toxic; c) they penetrate bone at sufficient concentrations to inhibit most members of the Enterobacteriaceae family, as well as a large percentage of Pseudomonas spp. and Staphylococcus strains. Quinolones were also used in combination with rifampin for prosthesis-related infections, with encouraging results ${ }^{[2]}$.

Rapid and efficient treatment is a necessary condition for avoiding therapeutical failures or relapsing infections. However to date, no antibiotic treatment, capable of being used for all the types of microorganisms found in chronic osteomyelitis, has been proposed for the treatment of chronic osteomyelitis.

In this work we present data on a three-year-long clinical study of chronic osteomyelitis treatment with either a standard or a specific therapy. We have found that standard therapy can be as efficacious as specific microbial-targeted therapy in curing osteomyelitis and avoiding relapses.

\section{MATERIALS AND METHODS}

Patients and treatment: 58 patients with chronic osteomyelitis were admitted to the II Orthopaedic Clinic of the University of Pisa, Italy. All of them had presented symptoms of bone infection for at least one month. The criteria for diagnosis and treatment were done according to previous studies ${ }^{[2,6,11]}$. The patients, after giving informed consent, were randomly divided into two groups: one (group A) received a standard therapy with the combination of a cephalosporin (generally $1 \mathrm{~g}$ ceftriaxone per day) and an aminoglycoside (150 $\mathrm{mg}$ netilmicin twice/day) administered intravenously for 15 days and subsequently an oral therapy for times ranging from 1 to 3 months with a fluoroquinolone $(250 \mathrm{mg}$ ciprofloxacin, norfloxacin or ofloxacin twice/day); the other (group B) received a specific therapy with various antibiotics, chosen according to the antibiogram performed on the bacterial strains isolated from the infection, consisting in parenteral treatment for 15 days and oral treatment for an additional 1-3 month period.

Microbiology: Wound material was obtained either during surgical debridement of bone infection or from skin drainage of the infection, when present. The sample treatment and the isolation and identification of the isolated strains were performed with standard methods ${ }^{[23]}$. Antibiograms were carried out with the Kirby-Bauer method on Muller-Hinton agar plates ${ }^{[23]}$. When needed, statistical evaluation was performed with the Student's t test.

\section{RESULTS}

Microorganisms isolated: 78 different microbial strains were isolated from 58 patients. In 44 cases the infections were monomicrobic; in 14 cases, 2 or more microorganisms were involved. The species of microorganisms found in the study are shown in Table 1. A comparison with the data from literature is also reported in the same table. In the present study the isolation of Staphylococcus aureus and Pseudomonas aeruginosa practically coincided with the data reported in the literature, whereas enterococci were isolated in a significantly lower amount. In 17 cases the patients showed one or more relapses of the infection after a course of therapy and a new antibiotic treatment was started (data not shown).

Sensitivity of the isolated strains to antibiotics: Table 2 shows the sensitivity of the most frequently bacterial strains isolated in this study, to ceftriaxone, netilmicin and ciprofloxacin. No strain of P. aeruginosa was resistant to any of these antibiotics, whilst $\mathrm{S}$. aureus was resistant to ceftriaxone in 3 cases and to netilmicin in 1 case; 1 strain of $\mathrm{S}$. epidermidis was resistant to ceftriaxone and 2 strains to netilmicin; no strain of Sthaphylococcus was resistant to ciprofloxacin. No bacterial strain was resistant to ceftriaxone and netilmicin used in combination. 
Table 1: Species and number of microorganisms isolated from chronic osteomyelitis in this study as compared to bacterial pathogens described in the literature, * [2,28] ( Rissing 1997; Emori 1993); ** Other microbes: Corynebacteria, Viridans streptococci, Peptococcus spp., Nutritionally variant streptococci.

\begin{tabular}{|c|c|c|c|c|}
\hline \multirow{2}{*}{ Species } & \multicolumn{2}{|c|}{ Isolated in this study } & \multicolumn{2}{|c|}{ Data in literature * } \\
\hline & No. & (\%) & No. & $(\%)$ \\
\hline Staphylococcus aureus & 21 & $(26.9)$ & 19 & $(25.3)$ \\
\hline Coagulase-negative Staphylococcus & 18 & $(23.0)$ & 14 & $(18.6)$ \\
\hline Pseudomonas aeruginosa & 9 & $(11.5)$ & 8 & $(10.6)$ \\
\hline $\begin{array}{c}\text { Other non-fermenting gram- } \\
\text { negatives }\end{array}$ & 5 & $(6.3)$ & 1 & $(1.3)$ \\
\hline Enterobacteria & 16 & $(21.0)$ & 20 & $(26.5)$ \\
\hline Enterococcus spp. & 2 & $(3.0)$ & 12 & $(16.0)$ \\
\hline Other microorganisms $^{* *}$ & 7 & $(10.2)$ & 1 & $(1.3)$ \\
\hline
\end{tabular}

Table 2: Susceptibility of the most frequently bacteria isolated in this study to some standard antibiotics

\begin{tabular}{|c|c|c|c|c|c|c|c|c|c|}
\hline \multirow{3}{*}{ Bacterial strains } & \multicolumn{6}{|c|}{$\begin{array}{l}\text { Antibiotic susceptibility } \\
\text { No. of strains }\end{array}$} & & & \\
\hline & \multicolumn{3}{|c|}{ Ceftriaxone } & \multicolumn{3}{|c|}{ Netilmicin } & \multicolumn{3}{|c|}{ Ciprofloxacin } \\
\hline & $\mathrm{S}$ & I & $\mathrm{R}$ & $\mathrm{S}$ & $\mathrm{I}$ & $\mathrm{R}$ & $\mathrm{S}$ & $\mathrm{I}$ & $\mathrm{R}$ \\
\hline Pseudomonas aeruginosa & 4 & 1 & 0 & 5 & 0 & 0 & 4 & 1 & 0 \\
\hline Staphylococcus aureus & 10 & 0 & 3 & 12 & 0 & 1 & 11 & 1 & 0 \\
\hline CN Staphylococci ${ }^{* *}$ & 3 & 1 & 1 & 4 & 0 & 2 & 6 & 0 & 0 \\
\hline
\end{tabular}

*S = susceptible, I = intermediate; $\mathrm{R}$ = resistant; **CN Staphylococci: coagulase-negative staphylococci

Therapeutic treatment: In Table 3, the results of the antibiotic treatment of the patients are shown, with the score of the 1 year follow-up after the end of therapy. Results showed that there was no significant difference between group A (treated with standard therapy) and group B ( treated with specific therapy). In the first case 25 patients resulted "clinically cured" ( $95.5 \%)$, while in the second group 29 patients (93.5\%) were cured. The difference was statistically non-significant.

The number of relapsed was quite closed in two groups (6 in the group A and 9 in the group B), but they were all cured after an additional (or 2 additional) course(s) of therapy .

Follow-up of patients with orthopaedic prosthesis: 9 patients bearing a bone prosthesis were included in the two groups. Most of them (8) were included in group A, whilst only 1 was in group B due to the impossibility of identifying the etiologic agent of the infection before starting the therapy; 5 of the patients were cured after a single antibiotic treatment cycle, whilst 4 patients required 2 or more therapy courses (Table 4). Since only 1 patient was included in group B, no significant evaluation of the different response to the therapy regimens could be detected for the two groups.

Table 3: one year follow up of 58 patients with cronich osteomyelitis treated with either standard therapy or specific terapy.

\begin{tabular}{ccccc}
\hline & \multicolumn{2}{c}{$\begin{array}{c}\text { Standard } \\
\text { therapy }\end{array}$} & \multicolumn{2}{c}{ Specific therapy } \\
& No. & $(\%)$ & No. & $(\%)$ \\
\hline Total No. of patients & 27 & $(46)$ & 31 & $(54)$ \\
58 & & & & \\
$\begin{array}{c}\text { Improvement after } 6 \\
\text { months }\end{array}$ & $\begin{array}{r}(85.1) \\
\text { Clinically cured after }\end{array}$ & 25 & 29 & $(80.6)$ \\
12 month follow-up & $(95.5)$ & & \\
Relapses & 6 & 9 & $(29.0)$ \\
Relapses cured after & $(22.1)$ & & \\
$\begin{array}{c}\text { additional courses (1-3) } \\
\text { of therapy }\end{array}$ & 6 & 9 & \\
\hline
\end{tabular}




\section{DISCUSSION}

To date, most clinicians and microbiologists have not reached unanimous consent on the best way of treating chronic osteomyelitis in terms of clinical and microbiological diagnosis, the means and duration of therapy, and antibiotic treatment. The etiologic agents are often variable and definite microbiological diagnosis and care are difficult to establish ${ }^{[22]}$. In

Table 4: Result of either specific or standard therapy on prosthesis-bearing patients

\begin{tabular}{|c|c|c|c|c|}
\hline Patient & $\begin{array}{c}\text { Microorganism } \\
\text { isolated }\end{array}$ & $\begin{array}{l}\text { Type of } \\
\text { therapy }\end{array}$ & $\begin{array}{l}\text { No. of } \\
\text { antibiotic } \\
\text { courses } \\
\text { performed }\end{array}$ & $\begin{array}{c}\text { Follow-up } \\
\text { after } 12 \\
\text { months }\end{array}$ \\
\hline $\mathrm{RG}$ & S. aureus & Standard & 4 & $\begin{array}{l}\text { Clinically } \\
\text { cured }\end{array}$ \\
\hline RI & S. aureus & Standard & 2 & $\begin{array}{l}\text { Clinically } \\
\text { cured }\end{array}$ \\
\hline PF & S. aureus & Standard & 4 & $\begin{array}{l}\text { Clinically } \\
\text { cured }\end{array}$ \\
\hline MGC & S. aureus & Standard & 2 & $\begin{array}{c}\text { Clinically } \\
\text { cured }\end{array}$ \\
\hline BL & Anaerobes & Specific & 1 & $\begin{array}{l}\text { Clinically } \\
\text { cured }\end{array}$ \\
\hline ST & S. epidermidis & Standard & 1 & $\begin{array}{l}\text { Clinically } \\
\text { cured }\end{array}$ \\
\hline $\mathrm{BZ}$ & $\begin{array}{c}\text { S. aureus }+ \\
\text { Enterobacteria }\end{array}$ & Standard & 1 & $\begin{array}{l}\text { Clinically } \\
\text { cured }\end{array}$ \\
\hline LBM & S. epidermidis & Standard & 1 & $\begin{array}{l}\text { Clinically } \\
\text { cured }\end{array}$ \\
\hline SG & S. epidermidis & Standard & 1 & $\begin{array}{l}\text { Clinically } \\
\text { cured }\end{array}$ \\
\hline
\end{tabular}

addition, the presence of foreign material complicates the treatment of osteomyelitis, often leading to the failure of therapy ${ }^{[22]}$. Although some authors ${ }^{[24,25]}$ have claimed that the appropriate diagnosis of chronic osteomyelitis is strictly dependent on microbiologic cultures of the infected bone, information about surgical treatment for osteomyelitis has not been provided by most clinicians in many recent studies. Furthermore bone debridement or biopsies for microbial analysis were only performed in a small number of studies and in many of the latest studies, the criteria for diagnosis and treatment were not clearly defined. Therefore, Lazzarini et $\mathrm{al}^{[26]}$ concluded that well designed and complete comparative studies to elucidate the most appropriate diagnostic and therapeutic regimen, are still lacking.

In this paper we present evidence that a standard therapy, which could be effective on most types of bacteria and could also be started before receiving the microbiology laboratory response, resulted as being at least as effective as a specific therapy, based on an antibiotic choice guided by the antibiogram assay. This standard therapy was quite effective in covering the most important bacteria usually found in chronic osteomyelitis, namely $\mathrm{S}$. aureus and P. aeruginosa, as well as other staphylococci and other Gram negative bacteria. There are several cases in which specific targeted therapy is difficult to start immediately. These can be: i) when surgical treatment of the infected bone is required before the laboratory analyses have been completed; ii) when various bacteria of different species are isolated from the infected fistula or from the patient's bone; iii) when no surgery or bone biopsy has been carried out and skin drainage is not present, as well as in all those cases where performing reliable microbiological analysis of the infected bone results as being difficult or even impossible.

The safety of prolonged use of the various antimicrobial agents and the cost and feasibility of the therapeutic regimen should be the parameters for a rational choice of the antibiotic ${ }^{[26]}$. Furthermore, the expense of long-regimen therapy should be considered and kept as low as possible ${ }^{[22]}$. Oral antimicrobial administration seems to be the best way to reduce costs and medical complications ${ }^{[22]}$.Oral quinolones were claimed to be as effective as standard parenteral therapy; thus oral therapy was proposed as an accepted alternative for selected patient treatment [22]. A minimum of 4-6 weeks of parenteral antimicrobial therapy targeting the causative organism, in conjunction with surgical debridement, has been proposed as the standard treatment for chronic longbone osteomyelitis in adults ${ }^{[22]}$. Parenteral therapy consisting of a semi-synthetic penicillin, clindamicin and an aminoglycoside, singly or in combination were considered as being a good regimen ${ }^{[9,27]}$. In the present study a very simple and relatively low cost therapy regimen is proposed, consisting of up to 15 days of standard parenteral antibiotic treatment with a broad spectrum cephalosporin, hospitalization was kept at a minimum and oral therapy could be performed at home.

Some authors have claimed that no significant differences in therapeutic efficacy were found in trials comparing oral fluoroquinolones with intravenous betalactam drugs as regards long term results ${ }^{[12]}$. They also stated that there is little high quality evidence on the relative effectiveness of the various regimens of antibiotic therapy for osteomyelitis ${ }^{[12]}$. In our experience, a short course (15 days) of parenteral broad-spectrum antibiotics was well tolerated and gave the most successful results, when it was followed by 1 to 3 months of treatment with an oral fluoroquinolone. The compliance was excellent and the follow-up after one year resulted as being a success for at least $95 \%$ of the patients treated, also including the prosthesis- 
bearing patients. Additional courses (from 1 to 3 ) of therapy in patients with persistent or relapsing infections were only needed in a small number of cases In conclusion, standard therapy performed with antibiotics which have shown an excellent activity on the most frequently isolated bacteria from chronic osteomyelitis, provides the physician with the chance of starting early antibiotic treatment on the admitted patients, in all those cases where a precise and definite diagnosis and a specific therapy, targeted on the isolated etiologic agents, is delayed or cannot be performed. This regimen can be used whenever possible, whilst waiting for a complete laboratory diagnosis, or as a definite treatment with an optimal prediction of a successful follow-up.

\section{ACKNOWLEDGEMENTS}

This work was supported by a local project ex $60 \%$ grant from the University of Cagliari.

\section{REFERENCES}

1. Guglielmo, B.J., A.D. Luber, D. Palotta and R.A. Jacobs, 2000. Ceftriaxone therapy for staphylococcal osteomyelitis: a review. Clin. Infect. Dis., 30: 205-207.

2. Rissing, J.P., 1997. Antimicrobial therapy for chronic osteomyelitis in adults: role of the quinolones. Clin. Infect. Dis., 25:1327-1333.

3. Carek, P.J., L.M. Dickerson and J.L. Sack, 2001. Diagnosis and management of osteomyelitis. Am. Fam. Physician, 63: 2413-2420.

4. Ellington, J.K., M. Harris, M.C. Hudson, S. Vishin, L.X. Webb and R. Sherertz, 2006. Intracellular Staphylococcus aureus and antibiotic resistance: implications for treatment of staphylococcal osteomyelitis. J. Orthop. Res., 24: 87-93.

5. Lew, D.P., and F.A. Waldvogel, 1997. Osteomyelitis. N. Engl. J. Med., 336: 999-1007.

6. Mader, J.T., G.C. Landon and J. Calhoun, 1993. Antimicrobial treatment of osteomyelitis. Clin Orthop., 295: 87-95.

7. Lew, D.P. and F.A. Waldvogel, 1999. Use of quinolones in osteomyelitis and infected orthopaedic prosthesis. Drugs, 56 (suppl.2): 85-91.

8. Museru, L.M. and C.N. Mcharo, 2001. Chronic osteomyelitis: a continuing challenge in developing countries. Int. Orthop., 25: 127-131.

9. Mader, J.T., M.E. Shirtliff, S.C. Bergquist and J. Calhoun, 1999. Antimicrobial treatment of chronic osteomyelitis. Clin. Orthop., 360: 47-65.
10. Uckay, I., M. Assal, L. Legout, P. Rohner, R. Stern, D. Lew et al., 2006. Recurrent osteomyelitis caused by infection with different bacterial strains without obvious source of infection. J. Clin. Microbiol., 44: 1194-1196.

11. Mader, J.T., C. Norden, J.D. Nelson and G.B. Calandra, 1992. Evaluation of new antiinfective drugs for the treatment of osteomyelitis in adults. Clin. Infect. Dis., 15: S155-161.

12. Stengel, D., K. Bauwens, J. Sehouli, A. Ekkernkamp and F. Porzsolt, 2001. Systematic review and meta-analysis of antibiotic therapy for bone and joint infection. Lancet Infect. Dis., 1: 175-188.

13. Trujillo, I.Z., G. Valladares and A. Nava, 1993. Ciprofloxacin in the treatment of chronic osteomyelitis in adults. Drugs, 45 (suppl. 3): 454455.

14. Peterson, L.R., L.M. Lissack, K. Canter, M.T. Fasching, C. Clabots and D.N. Gerding, 1989. Therapy of lower extremity infections with ciprofloxacin in patients with diabetes mellitus, peripheral vascular disease, or both. Am. J. Med., 86: 801-808.

15. Dan, M., Y. Siegman-Igra, S. Pitlik and R. Raz, 1990. Oral ciprofloxacin treatment of Pseudomonas aeruginosa osteomyelitis. Antimicrob. Agents Chemother., 34: 849-852.

16. Gentry, L.O. and G. Rodriguez-Gomez, 1990. Oral ciprofloxacin compared with parenteral antibiotics in the treatment of osteomyelitis. Antimicrob. Agents Chemother., 34: 40-43.

17. Gomis, M., J. Barberan and J. Lopez-Arceo, 1993. Pefloxacin in the treatment of osteomyelitis. Drugs, 45 (suppl 3): 456-457.2.

18. Gomis, M., J. Barberan, B. Sanchez, S. Khorrami, J. Borja and J. Garcia-Barbal, 1999. Oral ofloxacin versus parenteral imipenem-cilastatin in the treatment of osteomyelitis. Rev. Esp. Quimioter., 12: 244-249.

19. Greenberg, R.N., M.T. Newman, S. Shariaty and R.W. Pectol, 2000. Ciprofloxacin, Lomefloxacin, or Levofloxacin as treatment for chronic osteomyelitis. Antimicrob. Agents Chemother., 44:164-166

20. Greenberg, R.N., A.D. Tice and P.K. Marsh, 1987. Randomized trial of ciprofloxacin compared with other antimicrobial therapy in the treatment of osteomyelitis. Am. J. Med., 82: 266-269.

21. Nicolau, D.P., L. Nie, P.R. Tessier, H.P. Kourea and C.H. Nightingale, 1998. Prophylaxis of acute osteomyelitis with absorbable ofloxacinimpregnated beads. Antimicrob. Agents Chemother., 42: 840-842.

22. Shuford, J.A. and J.M. Steckelberg, 2003. Role of oral antimicrobial therapy in the management of osteomyelitis. Curr. Opin. Infect. Dis., 16: 515519. 
23. Reissmer, B.S., G.L. Woods, R.B. Jr. Thomson, D.H. Larone, L.S. Garcia and R.Y. Simisu, 1999. Specimen processing. In Murray P.R., Baron E.J., Pfaller M.A., Tenover F.C. and Yolken R.H. (Eds), Manual of Clinical Microbiology, 7th ed., pp 64104, ASM Press, Washington DC.

24. Salvana, J., C. Rodner, B.D. Bowner, K. Livingstone, J. Schreiber, E. Pesanti, 2005. Chronic osteomyelitis: results obtained by an integrated team approach to management. Conn. Med., 69: 195-202.

25. Zuluaga, A.F., W. Galvis, J.G. Saldarriaga, M. Agudelo, B.E. Salazar and O. Vesga, 2006. Etiologic diagnosis of chronic osteomyelitis: a prospective study. Arch. Intern. Med. 166: 95- 100.
26. Lazzarini, L., B.A. Lopsky and J.T. Mader, 2005. Antibiotic treatment for osteomyelitis: what have we learned from 30 years of clincal trials? Int. J. Infect. Dis., 9: 127-137.

27. Gentry, L.O. and G. Rodriguez-Gomez, 1991. Ofloxacin versus parenteral therapy for chronic osteomyelitis. Antimicrob. Agents Chemother., 35: 538-541.

28. Emori, T.G. and R.P.Gaynes, 1993. An overview on nosocomial infections, including the role of microbiology laboratory. Clin. Microbiol. Rev., 6: 428-442.of Klebsiella pneumoniae and Serratia marcescens. Infection, 11(6): 315-7. 